\title{
Harnessing Control of Radiolysis during Liquid Cell Electron Microscopy to Enable Visualization of Nanomaterial Transformation Dynamics
}

\author{
Taylor J. Woehl ${ }^{1}$ and Patricia Abellan ${ }^{2,3}$ \\ 1. University of Maryland, Department of Chemical and Biomolecular Engineering, College Park, \\ Maryland, U.S.A. \\ 2. SuperSTEM Laboratory, SciTech Daresbury Campus, Daresbury, U.K. \\ 3. Institute for Materials Research, School of Chemical and Process Engineering, University of Leeds, \\ Leeds, U.K.
}

The radiolysis-induced reduction of dissolved metallic precursor ions or complexes yields formation of metal nanocrystals due to fast reduction reaction kinetics of the precursor with reducing radicals [1]. Liquid cell electron microscopy (LCEM) has offered the opportunity to directly observe radiolysisinduced nanoparticle nucleation and growth via high energy electron $(200-300 \mathrm{keV})$ irradiation of precursor solutions and simultaneous in situ (scanning) transmission electron microscopy ((S)TEM) imaging [2]. However, high electron dose rates associated with (S)TEM imaging result in large yields of both reducing and oxidizing radicals, which leads to uncontrolled synthesis conditions in which there are several competing reactions associated with the formation of metal nanocrystals. To simplify reaction conditions and facilitate quantitative understanding of the reaction kinetics for nanomaterial synthesis, the large pool of radicals formed during radiolysis should be converted into either net reducing or net oxidizing conditions. To this end, we have recently identified a number of reaction environment control parameters for controlling nanomaterial transformation during LCEM, which broadly fall into the categories of electron beam control and chemical control [3].

Here we show that the reaction environment during LCEM can be fine-tuned using a number of solution chemistry and electron beam parameters (Table I). Solution chemistry parameters include radical scavengers, solution $\mathrm{pH}$, and solvent type. Radical scavengers are solutes that act on specific radical species, reacting quickly with these species and eliminating them from the reaction environment. Having a well-defined reaction environment without significant back reactions is highly desirable for synthesis of nanomaterials, especially for understanding fundamental nucleation and growth mechanisms. One specific example is the use of $\mathrm{N}_{2} \mathrm{O}$ to scavenge strongly reducing aqueous electrons to establish a more oxidizing environment containing predominantly hydroxyl radicals. Conversely, alcohols such as isopropanol or ethanol specifically scavenge oxidizing hydroxyl radicals, allowing for a purely reducing environment containing aqueous electrons and hydrogen radicals [4]. Solvent type strongly affects the types and concentrations of radicals formed. For instance, aromatic solvents are relatively resistant to radiation damage and have low radiation yields, which is advantageous for controlling the growth of nanomaterials.

Electron beam parameters include beam current, electron energy, and imaging mode. Rather than controlling the type of radicals formed, these parameters mainly control the amount and location of radicals created during LCEM experiments. Imaging mode (TEM or STEM) has an interesting effect on nucleation and growth of nanoparticles, where empirical evidence suggests that the point electron excitations in STEM quickly exceed the supersaturation condition needed for nucleation to occur, compared to the broad parallel illumination of TEM [5]. We expect these control parameters will provide researchers with a useful toolkit for designing general chemistry and materials science LCEM 
experiments by "directing" the effect of the electron beam to understand fundamental nanomaterial transformation mechanisms as well as minimize radiation damage to samples [6].

\section{References:}

[1] J Belloni, Catalysis Today 113 (2006), pp. 141-156.

[2] TJ Woehl et a., ACS Nano 6 (2012), pp. 8599-8610.

[3] TJ Woehl and P Abellan, Journal of Microscopy (2016), Submitted.

[4] E Sutter et al, Nature Communications 5 (2014).

[5] P Abellan et al, Chemical Communications 50 (2014), pp. 4873-4880.

[6] SuperSTEM is the UK EPSRC National Facility for Aberration-Corrected STEM, supported by the Engineering and Physical Science Research Council.

Table I. Summary of electron beam and solution chemistry parameters for control of LCEM reaction conditions.

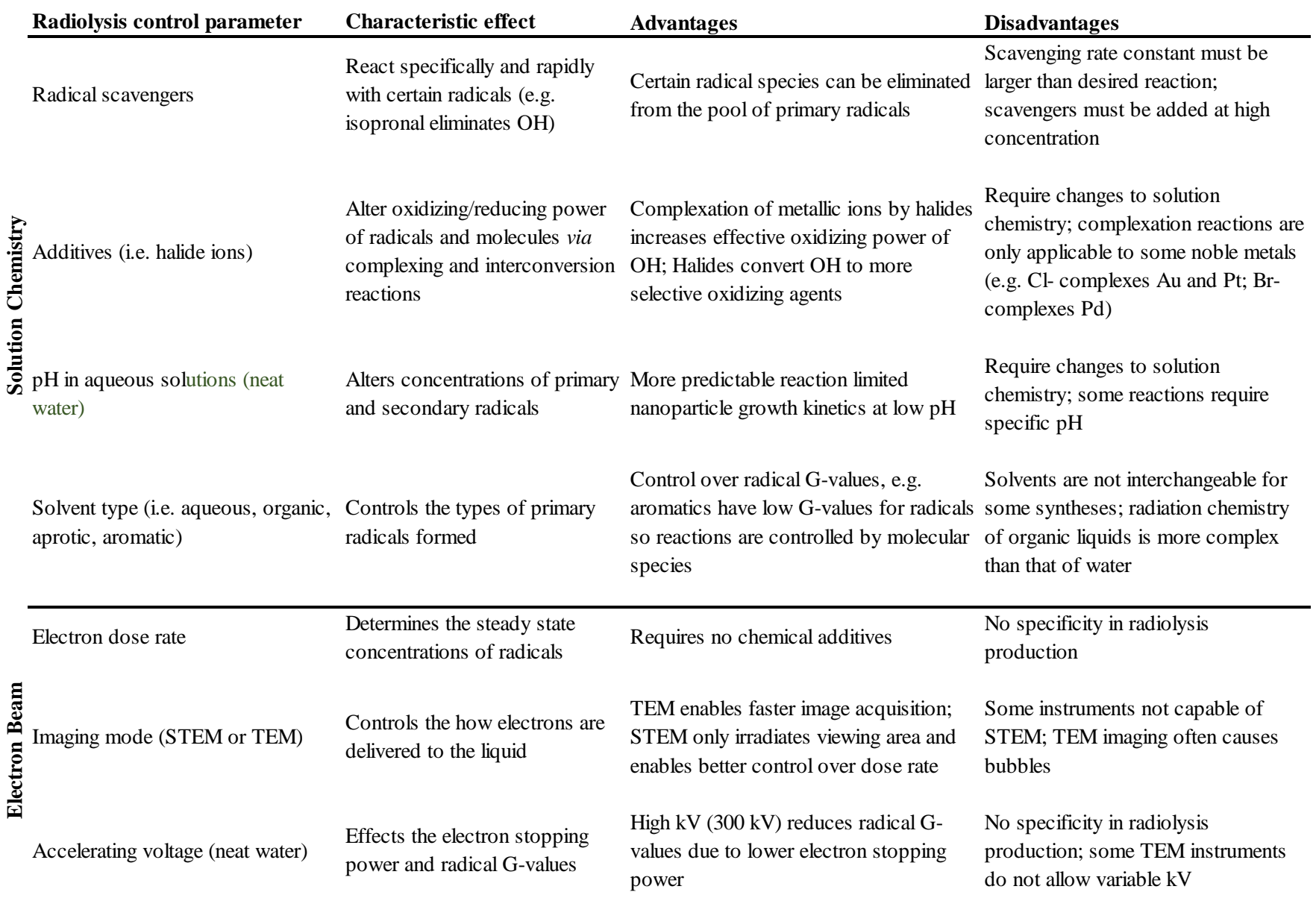

